\title{
A CONSISTENT ESTIMATOR FOR SPECTRAL DENSITY MATRIX OF A DISCRETE TIME PERIODICALLY CORRELATED PROCESS
}

BY

MAJID AZIMMOHSENI* (GORGAN), AHMAD REZA SOLTANI (SAFAT), MAHNAZ KHALAFI (GORGAN), AND NAEEMEH AKBAR I GHALESARY (GoRgAN)

Abstract. In this article, we introduce a weighted periodogram in the class of smoothed periodograms as a consistent estimator for the spectral density matrix of a periodically correlated process. We derive its limiting distribution that appears to be a certain finite linear combination of Wishart distribution. We also provide numerical derivations for our smoothed periodogram and exhibit its asymptotic consistency using simulated data.

2010 AMS Mathematics Subject Classification: Primary: 62M10; Secondary: 62M15.

Key words and phrases: Periodically correlated processes, spectral density matrix, Kullback-Leibler distance, smoothed periodogram, Wishart distribution, limiting distribution.

\section{INTRODUCTION}

A univariate zero mean second order process $\left\{X_{t} ; t \in \mathbb{Z}\right\}$ with covariance function $R(t, s)$ is called periodically correlated, $\mathrm{PC}$ in short, if

$$
R(t, s)=R(t+T, s+T), \quad t, s \in \mathbb{Z},
$$

for some integer $T>0$. The smallest such $T$ is defined to be a period of the process. The spectral density matrix of the process, $\mathbf{f}(\theta)=\left[f_{k-j}\left(\theta+\frac{2 \pi j}{T}\right)\right]_{j, k=0, \ldots, T-1}$, if exists, is a non-negative definite matrix-valued function. In this case,

$$
R(t, s)=\sum_{d=-T+1}^{T-1} \int_{0}^{2 \pi} e^{-i t \theta+i s(\theta+2 \pi d / T)} f_{d}(\theta) \mu(d \theta),
$$

where $f_{d}(\theta), d=-T+1, \ldots, T-1$, are called the spectral components of $\mathbf{f}$, see Gladyshev [2]. The literature on PC or cyclostationary processes is quite rich.

\footnotetext{
* Corresponding author.
} 
Therefore, we put light on what is done on spectral density estimation, which we discuss in this article. Nematollahi and Rao [6] derive a consistent estimator for the spectral density matrix of a PC process using the eigenvalue decomposition of block Toeplitz matrices. Hurd and Miamee [3] give a bivariate periodogram for a PC sequence to estimate spectral components of the spectral density matrix. Soltani and Azimmohseni [ [7], classically, introduce a periodogram matrix, then by using the Cholesky decomposition of spectral density matrix obtain the asymptotic distribution of the periodogram.

In the classical time series it is acknowledged that weighted periodograms for stationary processes are asymptotically consistent, and their variance goes to zero relatively fast. The rate of convergence is also specified. In this article we are concerned with weighted periodograms for periodically correlated processes that form a class of nonstationary processes rich in theory and practice. We introduce a class of weighted periodograms, derive rate of convergence for the variances and give their limiting distributions. This paper is organized as follows.

In Section 2, we provide some important spectral characterizations of PC processes including their spectral representations. We give the periodogram and its asymptotic properties. In Section 3, we derive interesting formulas for the covariance of the periodogram at different Fourier frequencies. In Section 4, we present a smoothed periodogram as an asymptotically consistent estimator for spectral density matrix of a PC process, and give the rate of the convergence. Finally, we establish the limiting distribution of the smoothed periodogram.

\section{SPECTRAL CHARACTERIZATIONS OF PC PROCESSES}

There are various spectral representations for PC processes. Gladyshev [2] views a PC process as the Fourier transform of a random measure with certain values dependencies, see also Hurd and Miamee [3]. Soltani and Shishebor [8] suggested an alternative representation: to view a PC process as a process with time dependent spectrum, namely

$$
X_{t}=\int_{0}^{2 \pi} e^{-i t \theta} V_{t}(\theta) \Phi(d \theta),
$$

where $V_{t}(\theta), t \in \mathbb{Z}, \theta \in[0,2 \pi)$, is a kernel which is $T$-periodic in $t ; V_{t+T}(\theta)=$ $V_{t}(\theta), \theta \in[0,2 \pi), t \in \mathbb{Z}$; and $\Phi$ is a random measure with orthogonal increment having finite spectral measure $\mu(d \theta)=E|\Phi(d \theta)|^{2}$ on $[0,2 \pi)$.

Let us define the process $Y_{t}$ by

$$
Y_{t}=\int_{0}^{2 \pi} e^{-i t \theta} \Phi(d \theta), \quad t \in \mathbb{Z}
$$

Then $Y_{t}$ is a purely random process on $\mathbb{Z} ; E Y_{t} \bar{Y}_{s}=0, t \neq s, E\left|Y_{t}\right|^{2}=1$. 
We let

$$
\mathbf{f}(\theta)=A(\theta) A^{*}(\theta)
$$

where

$$
A(\theta)=\left\|a_{j-k}\left(\theta+\frac{2 \pi j}{T}\right)\right\|_{j \geqslant k, j, k=0, \ldots, T-1}
$$

denotes the Cholesky factor of the spectral density $\mathbf{f}(\theta)$, where $a_{k}$ are complexvalued functions on $[0,2 \pi)$, subject to

$$
a_{0}(\theta)>0, \quad a_{k}(\theta)=0, \quad \theta \in[0,2 \pi k / T), k=0, \ldots, T-1, \text { a.e. } \theta,
$$

so that

$$
\begin{aligned}
& \sum_{k=0}^{T-1-d} a_{k}(\theta) \overline{a_{k+d}\left(\theta+\frac{2 \pi d}{T}\right)}=f_{d}(\theta), \quad d=1, \ldots, T-1, \\
& \begin{aligned}
\sum_{k=0}^{T-1}\left|a_{k}(\theta)\right|^{2} & =f_{0}(\theta), \\
\sum_{k=-d}^{T-1} a_{k}(\theta) a_{k+d}\left(\theta+\frac{2 \pi d}{T}\right) & =f_{d}(\theta), \quad d=-T+1, \ldots,-1 .
\end{aligned}
\end{aligned}
$$

A method to construct the kernel $V_{t}(\theta)$ in (2. I) is through the Cholesky factor of the spectral density, as follows:

$$
V_{t}(\theta)=\sum_{k=0}^{T-1} e^{-i \frac{2 \pi k t}{T}} a_{k}\left(\theta+\frac{2 \pi k}{T}\right), \quad \theta \in[0,2 \pi) .
$$

For the inference on periodogram, we let $X_{0}, \ldots, X_{N-1}$ be a finite segment of the PC process $\left\{X_{t}, t \in \mathbb{Z}\right\}$. The $T$-variate Fourier transform at frequency $\lambda \in$ $[0,2 \pi / T)$ is defined as

$$
\mathbf{d}_{X}(\lambda)=\left(d_{X}(\lambda), d_{X}\left(\lambda+\frac{2 \pi}{T}\right), \ldots, d_{X}\left(\lambda+\frac{2 \pi(T-1)}{T}\right)\right)^{\prime},
$$

where $d_{X}(\lambda+2 \pi j / T)=N^{-1 / 2} \sum_{t=0}^{N-1} X_{t} e^{i t(\lambda+2 \pi j / T)}, \quad j=0, \ldots, T-1$.

It will be more convenient to write the periodogram in the matrix form:

$$
\mathbf{I}_{X}(\lambda)=\mathbf{d}_{X}(\lambda) \mathbf{d}_{X}^{*}(\lambda) .
$$

It is common to define the discrete Fourier transform and periodogram with respect to Fourier frequencies $\lambda_{k}=2 \pi k / N, k=0, \ldots, N-1$, as follows:

$$
\mathbf{d}_{X}(\lambda)=\mathbf{d}_{X}\left(\lambda_{k}\right) \quad \text { for } \lambda_{k} \leqslant \lambda<\lambda_{k+1}, k=0, \ldots, N-1,
$$


and similarly

$$
\mathbf{I}_{X}(\lambda)=\mathbf{I}_{X}\left(\lambda_{k}\right) \quad \text { for } \lambda_{k} \leqslant \lambda<\lambda_{k+1}, k=0, \ldots, N-1,
$$

see Brockwell and Davis [I]]. Note that, for a PC process, the Fourier frequencies belong to the interval $[0,2 \pi / T)$. Indeed:

(i) For $\lambda_{j} \in[0,2 \pi / T)$, the set of $\left\{\lambda_{j}, \lambda_{j}+2 \pi / T, \ldots, \lambda_{j}+2 \pi(T-1) / T\right\}$ is the set of Fourier frequencies in $[0,2 \pi)$.

(ii) Every Fourier frequency $\lambda_{k} \in[0,2 \pi)$ is uniquely represented as $\lambda_{k}=$ $\lambda_{j}+2 \pi u(k, j) / T$, where $\lambda_{j} \in[0,2 \pi / T)$.

(iii) If $\lambda_{i}, \lambda_{j}$ are two different Fourier frequencies in $[0,2 \pi / T)$, then $\lambda_{i}+$ $2 \pi k / T \neq \lambda_{j}+2 \pi s / T$ for all $k, s=0, \ldots, T-1$.

(iv) Every Fourier frequency in $[0,2 \pi / T)$ is represented as

$$
\lambda_{k}=\frac{2 \pi k}{N}, \quad k=0, \ldots, m-1 .
$$

(v) If $\lambda_{i}+\lambda_{j}=2 \pi / T$, then $\lambda_{i}+2 \pi p / T+\lambda_{j}+2 \pi s / T=2 \pi$ for $p+s=$ $T-1$. If $\lambda_{i}+\lambda_{j} \neq 2 \pi / T$, then $\lambda_{i}+2 \pi p / T+\lambda_{j}+2 \pi s / T \neq 2 \pi$.

We apply

$$
\tilde{X}_{t}^{N}=N^{-1 / 2} \sum_{p=0}^{N-1} e^{i t \lambda_{p}} V_{t}\left(\lambda_{p}\right) d_{Y}\left(\lambda_{p}\right)
$$

to approximate the random integral in the formula (2.1). We form the finite segment $\tilde{X}_{0}, \ldots, \tilde{X}_{N-1}$ using the observed segment $X_{0}, \ldots, X_{N-1}$. Let $\mathbf{d}_{\tilde{X}}(\cdot)$ be the $T$ variate Fourier transform of $\tilde{X}_{0}, \ldots, \tilde{X}_{N-1}$. Then we deduce that

$$
\mathbf{d}_{\tilde{X}}\left(\lambda_{j}\right)=\mathbf{A}\left(\lambda_{j}\right) \mathbf{d}_{Y}\left(\lambda_{j}\right),
$$

where $\mathbf{A}(\cdot)$ is the Cholesky factor of the spectral density $\mathbf{f}(\cdot)$, and the $T$-variate $\mathbf{d}_{Y}\left(\lambda_{j}\right)$ is defined as in (2.3) for the purely random series given in (2.2); note that $E\left(\mathbf{d}_{Y}\left(\lambda_{j}\right) \mathbf{d}_{Y}^{*}\left(\lambda_{j}\right)\right)=I_{T}$.

For the Fourier frequency $\lambda_{j} \in[0,2 \pi / T)$, the components of the vector $\mathbf{d}_{\tilde{X}}\left(\lambda_{j}\right)$ are given by

$$
d_{\tilde{X}}\left(\lambda_{j}+\frac{2 \pi s}{T}\right)=\sum_{k=0}^{T-1} a_{k}\left(\lambda_{j}+\frac{2 \pi s}{T}\right) d_{Y}\left(\lambda_{p(k, j, s)}\right),
$$

where $\lambda_{p(k, j, s)}$ is uniquely determined by $\lambda_{p(k, j, s)}=\lambda_{j}+2 \pi(s-k) / T$ for $s=$ $0, \ldots, T-1$. Moreover, the periodogram in terms of $\tilde{X}_{0}, \ldots, \tilde{X}_{N-1}$ is defined by

$$
\mathbf{I}_{\tilde{X}}\left(\lambda_{j}\right)=\mathbf{d}_{\tilde{X}}^{T}\left(\lambda_{j}\right) \mathbf{d}_{\tilde{X}}^{T}\left(\lambda_{j}\right)^{*}=\left[\tilde{I}_{p q}\left(\lambda_{j}\right)\right]_{p, q=0, \ldots, T-1},
$$


where

$$
\tilde{I}_{p q}\left(\lambda_{j}\right)=d_{\tilde{X}}\left(\lambda_{j}+\frac{2 \pi p}{T}\right) \overline{d_{\tilde{X}}\left(\lambda_{j}+\frac{2 \pi q}{T}\right)} .
$$

It is clear that

$$
E\left(\mathbf{I}_{\tilde{X}}\left(\lambda_{j}\right)\right)=\mathbf{f}\left(\lambda_{j}\right) .
$$

Moreover, if $\lambda_{1}<\ldots<\lambda_{J}$ are arbitrary Fourier frequencies in $(0,2 \pi / T)$, then $\mathbf{d}_{\tilde{X}}\left(\lambda_{1}\right), \ldots, \mathbf{d}_{\tilde{X}}\left(\lambda_{J}\right)$ are uncorrelated. If the white noise $\left\{Y_{t}, t \in Z\right\}$ is Gaussian, then these vectors are independent, and consequently, $\mathbf{I}_{\tilde{X}}^{T}\left(\lambda_{j}\right), j=1, \ldots, J$, are independent and distributed as $W_{T}^{C}\left(1, \mathbf{f}\left(\lambda_{j}\right)\right)$, where " $W_{T}^{C}(\cdot, \cdot)$ " stands for the complex Wishart distribution.

In order to investigate the asymptotic properties of the discrete Fourier transform and the periodogram of PC processes, we apply $\left\{\tilde{X}_{t}, t \in \mathbb{Z}\right\}$ as an auxiliary operator, as in Soltani and Azimmohseni [ $[$ ], where it is proved that under the condition that the Cholesky factor $\mathbf{A}(\cdot)$ of the spectral density matrix $\mathbf{f}(\cdot)$ is continuous, for arbitrary frequencies $\lambda_{1}<\ldots<\lambda_{J}$ in $(0,2 \pi / T), \mathbf{d}_{X}^{T}\left(\lambda_{1}\right), \ldots, \mathbf{d}_{X}^{T}\left(\lambda_{J}\right)$ are asymptotically independent and distributed as $N_{T}^{c}\left(0, \mathbf{f}\left(\lambda_{j}\right)\right), j=1, \ldots, J$. Moreover, $\mathbf{I}_{X}^{T}\left(\lambda_{j}\right)$ are asymptotically independent $W_{T}^{C}\left(1, \mathbf{f}\left(\lambda_{j}\right)\right)$ for $j=1, \ldots, J$. Using the auxiliary operator is somewhat new and is a short cut to the classical lengthy procedure of derivations of the periodogram asymptotic distribution given by Brockwell and Davis [T]].

\section{PERIODOGRAM COVARIANCES}

This section is devoted to the formulations for covariances between the periodogram at Fourier frequencies. The following kernels are basic tools, namely the Dirichlet kernel, the Fejér kernel and the generalized spectral kernel $S_{N}(\cdot ; \cdot, \cdot)$ introduced by Soltani and Azimmohseni [ [ 7 , given below.

Suppose $D_{N}(\theta)=\sum_{t=0}^{N-1} e^{i t \theta}, \theta \in[0,2 \pi)$, is the Dirichlet kernel, and let

$$
S_{N}\left(\theta ; \eta, \eta^{\prime}\right)=\frac{D_{N}(\theta-\eta) D_{N}\left(\theta-\eta^{\prime}\right)}{N}, \quad \theta \in[0,2 \pi), \eta, \eta^{\prime} \in[0,2 \pi) .
$$

Then $S_{N}\left(\theta ; \eta, \eta^{\prime}\right)$, as a function of $\theta$, has the following properties:

(i) $S_{N}(\theta ; \eta, \eta)=K_{N}(\theta-\eta)$, where $K_{N}$ is the Fejér kernel.

(ii) $S_{N}\left(\theta ; \eta, \eta^{\prime}\right) \rightarrow 0, N \rightarrow \infty$, for $\eta \neq \eta^{\prime}, \theta \in[0,2 \pi), \theta \neq \eta, \theta \neq \eta^{\prime}$.

(iii) For any $0<\delta<\frac{1}{2}\left|\eta-\eta^{\prime}\right|,\left|S_{N}\left(\theta ; \eta, \eta^{\prime}\right)\right|<1 / \sin ^{2}(\delta / 2), \quad N \geqslant 1, \theta \in$ $[0,2 \pi) ; \eta, \eta^{\prime} \in[0,2 \pi), \eta \neq \eta^{\prime}$.

(iv) If either $\theta-\eta$ or $\theta-\eta^{\prime}$ is a Fourier frequency in $(0,2 \pi)$, then $S_{N}\left(\theta ; \eta, \eta^{\prime}\right)$ $=0$; also, if both $\theta-\eta, \theta-\eta^{\prime} \in\{0,2 \pi\}$, then $S_{N}\left(\theta ; \eta, \eta^{\prime}\right)=N$.

The following lemma is a version of the classic result for the periodogram of white noise processes given by Brockwell and Davis [IT]. For notational conve- 
nience we let

$$
\begin{aligned}
& \sigma_{i j}^{(Y)}(p, q ; s, r) \\
= & \operatorname{cov}\left(d_{Y}\left(\lambda_{i}+\frac{2 \pi p}{T}\right) \overline{d_{Y}\left(\lambda_{i}+\frac{2 \pi q}{T}\right)}, d_{Y}\left(\lambda_{j}+\frac{2 \pi s}{T}\right) \overline{d_{Y}\left(\lambda_{j}+\frac{2 \pi r}{T}\right)}\right)
\end{aligned}
$$

denote the periodogram covariance of a process $\left\{Y_{t}, t \in \mathbb{Z}\right\}$.

LEMMA 3.1. Let $Y_{t}$ be a white noise process, let $d_{Y}(\cdot)$ denote the finite Fourier transform of the finite segment $Y_{0}, \ldots, Y_{N-1}$ and let $\lambda_{i}, \lambda_{j} \in(0,2 \pi / T)$. Then:

(i) For $\lambda_{i} \neq \lambda_{j}$ and $\lambda_{i}+\lambda_{j} \neq 2 \pi / T$,

$$
\sigma_{i j}^{(Y)}(p, q ; s, r)=O\left(N^{-1}\right) .
$$

(ii) For $\lambda_{i} \neq \lambda_{j}$ and $\lambda_{i}+\lambda_{j}=2 \pi / T$,

$$
\sigma_{i j}^{(Y)}(p, q ; s, r)= \begin{cases}1-O\left(N^{-1}\right), & p+s=q+r=T-1, \\ O\left(N^{-1}\right), & \text { otherwise. }\end{cases}
$$

(iii) For $\lambda_{i}=\lambda_{j}$,

$$
\sigma_{i i}^{(Y)}(p, q ; s, r)= \begin{cases}1+O\left(N^{-1}\right), & p=q=s=r \\ 1+O\left(N^{-1}\right), & (p=r) \neq(s=q), \\ O\left(N^{-1}\right), & \text { otherwise. }\end{cases}
$$

In the following we bring our formulations for the covariances of the periodogram of $\tilde{X}_{t}$ at Fourier frequencies.

THEOREM 3.1. Assume the spectral density $\mathbf{f}(\cdot)$ is positive definite and continuous. Then for $\lambda_{i}, \lambda_{j} \in[0,2 \pi / T)$ such that $\lambda_{i}+\lambda_{j} \neq 2 \pi / T$ and for all $p, q, s, r$ $=0,1, \ldots, T-1$,

$\sigma_{i j}^{(\tilde{X})}(p, q ; s, r)= \begin{cases}O\left(N^{-1}\right), & \lambda_{i} \neq \lambda_{j}, \\ f_{r-p}\left(\lambda_{i}+2 \pi q / T\right) \overline{f_{s-q}\left(\lambda_{i}+2 \pi p / T\right)}+O\left(N^{-1}\right), & \lambda_{i}=\lambda_{j},\end{cases}$ for all $p, q, s, r \in\{0,1, \ldots, T-1\}$.

COROLLARY 3.1. Assume $\mathbf{I}_{\tilde{X}}(\cdot)=\left[\tilde{I}_{p q}(\cdot)\right]_{p, q=0, \ldots, T-1}$ is the periodogram of $\left\{\tilde{X}_{0}, \ldots, \tilde{X}_{N-1}\right\}$. Then for all $\lambda_{i}, \lambda_{j} \in(0,2 \pi / T)$ such that $\lambda_{i}+\lambda_{j} \neq 2 \pi / T$ and for $p, q, r, s \in\{0, \ldots, T-1\}$,

$$
\begin{aligned}
\operatorname{cov}\left(\tilde{I}_{p q}\left(\lambda_{i}\right), \tilde{I}_{s r}\left(\lambda_{j}\right)\right) & \\
\quad & = \begin{cases}O\left(N^{-1}\right), & \lambda_{i} \neq \lambda_{j}, \\
f_{r-p}\left(\lambda_{i}+2 \pi q / T\right) \overline{f_{s-q}\left(\lambda_{i}+2 \pi p / T\right)}+O\left(N^{-1}\right), & \lambda_{i}=\lambda_{j} .\end{cases}
\end{aligned}
$$


COROLLARY 3.2. Special cases of Corollary 3.1 can be considered as follows:

$$
\begin{aligned}
\operatorname{var}\left(\tilde{I}_{p p}\left(\lambda_{i}\right)\right) & =f_{0}\left(\lambda_{i}+\frac{2 \pi p}{T}\right) \overline{f_{0}\left(\lambda_{i}+\frac{2 \pi p}{T}\right)}+O\left(N^{-1}\right) \\
& =f_{0}^{2}\left(\lambda_{i}+\frac{2 \pi p}{T}\right)+O\left(N^{-1}\right), \\
\operatorname{var}\left(\tilde{I}_{p q}\left(\lambda_{i}\right)\right)= & f_{q-p}\left(\lambda_{i}+\frac{2 \pi p}{T}\right) \overline{f_{p-q}\left(\lambda_{i}+\frac{2 \pi q}{T}\right)}+O\left(N^{-1}\right) .
\end{aligned}
$$

According to Corollaries 3.1 and 3.2, the periodogram is not a consistent estimator for spectral density of a PC process.

In the next section, we present a consistent estimator for spectral density by smoothing the periodogram matrix.

\section{ASYMPTOTICALLY CONSISTENT ESTIMATORS}

In order to construct a consistent estimator for the spectral density, we propose the following weighted (smoothed) periodogram:

$$
\mathbf{I}^{W}(\lambda)=\sum_{|k| \leqslant u_{n}} W_{n}(k) \mathbf{I}\left(\lambda+\lambda_{k}\right),
$$

where $\left\{u_{n}\right\}$ and $\left\{W_{n}(\cdot)\right\}$ are sequences of band-widths and weight functions, respectively. We use similar weight functions for all the elements of periodogram matrix. Technically, we need to impose the following assumptions on the weight functions $\left\{W_{n}(\cdot)\right\}$ and the band-width sequence $\left\{u_{n}, n \in \mathbb{Z}\right\}$ :

$$
u_{n} \rightarrow \infty \quad \text { and } \quad \frac{u_{n}}{N} \rightarrow 0 \quad \text { as } N \rightarrow \infty .
$$

Therefore, for all $k \leqslant u_{n}$ and for a fixed frequency $\lambda, \lambda+\lambda_{k}^{\left(u_{n}\right)} \rightarrow \lambda$ as $N \rightarrow \infty$.

$$
\begin{gathered}
W_{n}(k)=W_{n}(-k) \quad \text { and } \quad W_{n}(k) \geqslant 0 \quad \text { for all } k . \\
\sum_{|k| \leqslant u_{n}} W_{n}(k)=1 . \\
\sum_{|k| \leqslant u_{n}} W_{n}^{2}(k) \rightarrow 0 \quad \text { as } N \rightarrow \infty
\end{gathered}
$$

see Brockwell and Davis [I]]. In order to show the consistency of the weighted periodogram, we apply the weighted periodogram $\mathbf{I}_{\tilde{X}}^{W}(\lambda)=\left[\tilde{I}_{p q}^{w}(\lambda)\right]_{p, q=0, \ldots, T-1}$ in terms of $\left\{\tilde{X}_{0}, \ldots, \tilde{X}_{N-1}\right\}$ as follows:

$$
\mathbf{I}_{\tilde{X}}^{W}(\lambda)=\sum_{|k| \leqslant u_{n}} W_{n}(k) \mathbf{I}_{\tilde{X}}\left(\lambda+\lambda_{k}\right) .
$$


In particular, let us put $u_{n}=n$ and $W_{n}(k)=1 /(2 n+1)$ for $|k| \leqslant n$, where $n / N \rightarrow 0$ as $N \rightarrow+\infty$. Then it is easy to see that

$$
\mathbf{I}_{\tilde{X}}^{W}\left(\lambda_{j}\right)=\frac{1}{2 n+1} \sum_{|k| \leqslant n} \mathbf{I}_{\tilde{X}}\left(\lambda_{j}+\lambda_{k}\right)
$$

is distributed as $W_{T}^{C}\left(2 n+1, \mathbf{f}\left(\lambda_{j}\right) /(2 n+1)^{2}\right)$. This fact shows that the weighted periodogram is a consistent estimator for spectral density.

The following theorem is for the general case.

THEOREM 4.1. Suppose the spectral density $\mathbf{f}(\cdot)=\left[f_{p q}(\cdot)\right]_{p, q=0, \ldots, T-1}$ on $[0,2 \pi / T)$ is continuous. Then $\tilde{\mathbf{f}}(\cdot)=\mathbf{I}_{\tilde{X}}^{W}(\cdot)$ has the following properties:

(a) $E\left(\tilde{\mathbf{f}}\left(\lambda_{j}\right)\right)=\mathbf{f}\left(\lambda_{j}\right)$ for $\lambda_{j} \in[0,2 \pi / T)$.

(b) For all $\lambda_{i} \neq \lambda_{j} \in[0,2 \pi / T)$ and $p, q, s, r=0, \ldots, T-1$,

$$
\operatorname{cov}\left(\tilde{f}_{p q}\left(\lambda_{i}\right), \tilde{f}_{r s}\left(\lambda_{j}\right)\right) \rightarrow 0 \quad \text { as } N \rightarrow \infty .
$$

(c) For all $\lambda_{i} \in[0,2 \pi / T)$ and $p, q, s, r=0, \ldots, T-1$,

$$
\operatorname{cov}\left(\tilde{f}_{p q}\left(\lambda_{i}\right), \tilde{f}_{r s}\left(\lambda_{i}\right)\right) \rightarrow 0 \quad \text { as } N \rightarrow \infty .
$$

In particular, $\operatorname{var}\left(\tilde{f}_{p q}\left(\lambda_{i}\right)\right) \rightarrow 0$, and $\operatorname{var}\left(\tilde{f}_{p p}\left(\lambda_{i}\right)\right) \rightarrow 0$ as $N \rightarrow \infty$.

(d) For $\lambda_{i}, \lambda_{j} \in[0,2 \pi / T)$,

$$
\begin{array}{r}
\lim _{N \rightarrow \infty}\left(\sum_{|k| \leqslant u_{n}} W_{n}^{2}(k)\right)^{-1} \operatorname{cov}\left(\tilde{f}_{p q}\left(\lambda_{i}\right), \tilde{f}_{s r}\left(\lambda_{j}\right)\right) \\
\quad= \begin{cases}0, & \lambda_{i} \neq \lambda_{j}, \\
f_{r-p}\left(\lambda_{i}+2 \pi p / T\right) \overline{f_{s-q}\left(\lambda_{j}+2 \pi q / T\right)}, & \lambda_{i}=\lambda_{j} .\end{cases}
\end{array}
$$

(e) For $\lambda_{i} \in[0,2 \pi / T), \tilde{\mathbf{f}}\left(\lambda_{i}\right)$ has the same asymptotic distribution as a linear combination of independent complex Wishart matrices:

$$
\tilde{\mathbf{f}}\left(\lambda_{i}\right) \stackrel{d}{=} \sum W_{n}(k) U_{k},
$$

where $U_{k}$ is distributed as $W_{T}^{C}\left(1, \mathbf{f}\left(\lambda_{i+k}\right)\right)$.

Note that a linear combination of Wishart matrices with positive coefficients can be approximated with a Wishart distribution, see Tan and Gupta [9] and Khuri et al. [5]. Although the method has been obtained for real Wishart matrices, it can be effectively used for complex Wishart matrices with real positive coefficients. Therefore, the distribution of weighted periodogram in Theorem 4.1(e), for fixed $\lambda_{i} \in[0,2 \pi / T)$, can be approximated as $W_{T}^{C}\left(u, \mathbf{g}\left(\lambda_{i}\right)\right)$, where $u$ and $\mathbf{g}\left(\lambda_{i}\right)$ can be expressed in terms of $\mathbf{f}\left(\lambda_{i+k}\right),|k| \leqslant u_{n}$. Using similar notation to that in Khuri et 
al. [5], let $d_{i}^{*}(p q, s t)=\sum_{|k| \leqslant u_{n}} W_{n}^{2}(k) f_{p q}\left(\lambda_{i+k}\right) \overline{f_{s t}\left(\lambda_{i+k}\right)}$. Also, let $\mathbf{f}^{*}\left(\lambda_{i}\right)$ denote the $T^{*} \times T^{*}$ matrix, $T^{*}=\frac{1}{2} T(T+1)$, whose elements are $d_{i}^{*}(p q, s t)$ arranged in lexicographic order; that is, $d_{i}^{*}\left(p_{1} q_{1}, s_{1} t_{1}\right)$ appears before $d_{i}^{*}\left(p_{2} q_{2}, s_{2} t_{2}\right)$ in a row if $q_{2}>q_{1}$, or $q_{2}=q_{1}$ and $p_{2}>p_{1}$. Similarly, $d_{i}^{*}\left(p_{1} q_{1}, s_{1} t_{1}\right)$ is before $d_{i}^{*}\left(p_{2} q_{2}, s_{2} t_{2}\right)$ in a column if $t_{2}>t_{1}$, or $t_{2}=t_{1}$ and $s_{2}>s_{1}$. Now, $u$ and $\mathbf{g}\left(\lambda_{i}\right)$ are computed as follows:

$$
\begin{gathered}
u=\left(\frac{\left|\sum_{|k| \leqslant u_{n}} W_{n}(k) \mathbf{f}\left(\lambda_{i+k}\right)\right|^{T+1}}{\left|\mathbf{f}^{*}\left(\lambda_{i}\right)\right|}\right)^{1 / T^{*}}, \\
\mathbf{g}\left(\lambda_{i}\right)=\frac{1}{u} \sum_{|k| \leqslant u_{n}} W_{n}(k) \mathbf{f}\left(\lambda_{i+k}\right) .
\end{gathered}
$$

Let us now present the asymptotic properties of the actual estimator of spectral density matrix $\hat{\mathbf{f}}(\lambda)$, i.e.,

$$
\hat{\mathbf{f}}(\lambda)=\mathbf{I}_{X}^{W}(\lambda)=\sum_{|k| \leqslant u_{n}} W_{n}(k) \mathbf{I}_{X}\left(\lambda+\lambda_{k}\right) .
$$

Since the periodograms of the actual series $X_{1}, \ldots, X_{N}$ and the auxiliary series $\tilde{X}_{1}, \ldots, \tilde{X}_{N}$ have the same asymptotic distribution, the same results as in Theorem 4.1 can be achieved for the estimator (4.7).

THEOREM 4.2. Suppose the spectral density $\mathbf{f}(\cdot)=\left[f_{i j}(\cdot)\right]_{i=0, \ldots, T-1}^{j=0, \ldots, T-1}$ is continuous on $[0,2 \pi / T)$. Then $\hat{\mathbf{f}}(\cdot)=\mathbf{I}^{W}(\cdot)$ has the following properties:

(a) $E\left(\hat{\mathbf{f}}\left(\lambda_{j}\right)\right)=\mathbf{f}\left(\lambda_{j}\right)$ for $\lambda_{j} \in[0,2 \pi / T)$.

(b) For all $\lambda_{i} \neq \lambda_{j} \in[0,2 \pi / T)$ and $p, q, s, r=0, \ldots, T-1$,

$$
\operatorname{cov}\left(\hat{f}_{p q}\left(\lambda_{i}\right), \hat{f}_{r s}\left(\lambda_{j}\right)\right) \rightarrow 0 \quad \text { as } N \rightarrow \infty .
$$

(c) For all $\lambda_{i} \in[0,2 \pi / T)$ and $p, q, s, r=0, \ldots, T-1$,

$$
\operatorname{cov}\left(\hat{f}_{p q}\left(\lambda_{i}\right), \hat{f}_{r s}\left(\lambda_{i}\right)\right) \rightarrow 0 \quad \text { as } N \rightarrow \infty .
$$

In particular, $\operatorname{var}\left(\hat{f}_{p q}\left(\lambda_{i}\right)\right) \rightarrow 0$, and $\operatorname{var}\left(\hat{f}_{p p}\left(\lambda_{i}\right)\right) \rightarrow 0$ as $N \rightarrow \infty$.

(d) For $\lambda_{i}, \lambda_{j} \in[0,2 \pi / T)$,

$$
\begin{array}{r}
\lim _{N \rightarrow \infty}\left(\sum_{|k| \leqslant u_{n}} W_{n}^{2}(k)\right)^{-1} \operatorname{cov}\left(\hat{f}_{p q}\left(\lambda_{i}\right), \hat{f}_{s r}\left(\lambda_{j}\right)\right) \\
\quad= \begin{cases}0, & \lambda_{i} \neq \lambda_{j}, \\
f_{r-p}\left(\lambda_{i}+2 \pi p / T\right) \overline{f_{s-q}\left(\lambda_{j}+2 \pi q / T\right)}, & \lambda_{i}=\lambda_{j} .\end{cases}
\end{array}
$$


(e) For $\lambda_{i} \in[0,2 \pi / T), \hat{\mathbf{f}}\left(\lambda_{i}\right)$ has the same asymptotic distribution as a linear combination of independent complex Wishart matrices:

$$
\tilde{\mathbf{f}}\left(\lambda_{i}\right) \stackrel{d}{=} \sum W_{n}(k) U_{k},
$$

where $U_{k}$ is distributed as $W_{T}^{C}\left(1, \mathbf{f}\left(\lambda_{i+k}\right)\right)$.

\section{NUMERICAL RESULTS}

In this section we conduct a simulation study to illustrate the efficiency of the estimator (4.7). In order to make a comparison between the actual spectral density $\mathbf{f}(\lambda)$ and its estimator $\hat{\mathbf{f}}(\lambda)$, we utilize the distance measuring function

$$
D_{H}(\hat{\mathbf{f}} ; \mathbf{f})=\int_{0}^{2 \pi / T} H\left(\hat{\mathbf{f}}(\lambda) \mathbf{f}^{-1}(\lambda)\right) d \lambda
$$

for some matrix-valued function $H(\cdot)$. In order to have the symmetric property for our distance measuring function, we replace the function $H$ in (5.1) with

$$
\tilde{H}(\mathbf{Z})=H(\mathbf{Z})+H\left(\mathbf{Z}^{-\mathbf{1}}\right) .
$$

There are two commonly used functions $H$ :

$$
\begin{gathered}
H_{\mathbf{I}}(\mathbf{Z})=\operatorname{trace}(\mathbf{Z})-\log (|\mathbf{Z}|)-T, \\
H_{\alpha}(\mathbf{Z})=\log \left|\alpha \mathbf{Z}+(1-\alpha) \mathbf{I}_{T}\right|-\alpha \log |\mathbf{Z}|,
\end{gathered}
$$

where $|\cdot|$ stands for the determinant of a matrix. The distance measuring functions (5.2) and (5.3) are called Kullback-Leibler and Chernoff disparity measures, respectively, see Kakizawa et al. [4].

In practice, the integral in (5.D) is approximated by sum over Fourier frequencies at the interval $[0,2 \pi / T)$, i.e., $\lambda_{k}=2 \pi k / N, k=0, \ldots, m-1$, where $m=N / T$. By using $\tilde{H}(\cdot)$ and the estimator of spectral density, we obtain the following integral approximation:

$$
D_{\tilde{H}}(\hat{\mathbf{f}} ; \mathbf{f}) \approx \sum_{k=0}^{m-1} \tilde{H}\left(\hat{\mathbf{f}}\left(\lambda_{k}\right) \mathbf{f}^{-1}\left(\lambda_{k}\right)\right) .
$$

Let us give three examples to illustrate the asymptotic properties of the estimator (4.7). For each example we use the Daniell kernel to smooth the periodograms. Moreover, to calculate the average distance between the actual spectral density matrices and their corresponding estimators, we replicate the simulation 100 times.

EXAmple 5.1. Assume a zero mean PC process $\left\{X_{t}, t \in \mathbb{Z}\right\}$ is a $\operatorname{PAR}(1)$, $\operatorname{PCAR}(1)$, process:

$$
X_{k T+\nu}=\phi_{\nu} X_{k T+\nu-1}+e_{k T+\nu}, \quad k \in Z, \quad \nu=0, \ldots, T-1,
$$


with the spectral components $f_{k}(\theta)=g_{k}(\theta)$ for $\theta \in[0,2 \pi(T-k) / T)$, and $f_{-k}(\theta)$ $=g_{T-k}(\theta)$ for $\theta \in[2 \pi(T-k) / T, 2 \pi)$, where

$$
g_{k}(\theta)=\left|1-A e^{-i T \theta}\right|^{-2} \sum_{l=0}^{T-1} \hat{G}_{l}\left(\theta+\frac{2 \pi l}{T}\right) \overline{\hat{G}}_{l-k}\left(\theta+\frac{2 \pi l}{T}\right), \quad k=0, \ldots, T-1,
$$

in which

$$
\hat{G}_{j}(\theta)=\frac{1}{T} \sum_{n=0}^{T-1} G_{n}(\theta) e^{i 2 \pi j n / T}, \quad j \in \mathbb{Z},
$$

with $G_{n}(\theta)=\sum_{k=0}^{T-1} A_{n-k+1}^{n} e^{-i k \theta}, A_{r}^{s}=\prod_{j=r}^{s} \nu_{j}$ for $r \leqslant s, A_{r}^{s}=1$ for $r>s$, and $A=\prod_{j=0}^{T-1} \nu_{j}$. To evaluate the distance between the spectral density matrix and its estimator in this example, we take exactly the same data as Nematollahi and Rao [6].

Figure 1 shows the average distance for different choices of sample size.

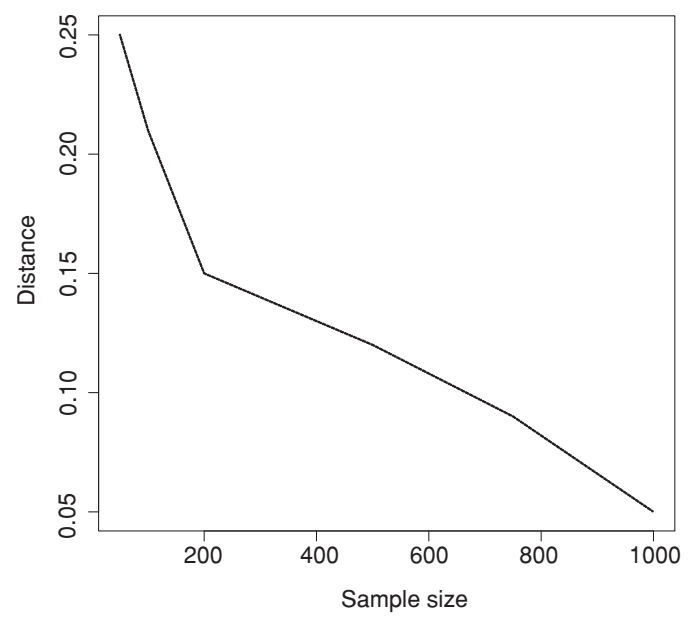

FiguRE 1. The Kullback-Leibler distance between actual spectral density matrix of the process (5.5) and its estimate for different choices of sample size.

EXAMPLE 5.2. Assume a zero mean PC process $\left\{X_{t}, t \in \mathbb{Z}\right\}$ to be $\operatorname{PCMA}(2)$, with the following structure:

$$
X_{k T+\nu}=Z_{k T+\nu}+\cos (\nu) Z_{k T+\nu-1}+\sin (\nu) Z_{k T+\nu-2}, \quad k \in Z, \quad \nu=0, \ldots, T-1 .
$$

For $T=2$ the spectral density matrix can be expressed as

$$
\mathbf{f}(\theta)=2 D(2 \theta) \mathbf{h}(2 \theta) D^{*}(2 \theta), \quad \theta \in[0, \pi),
$$

where

$$
D(2 \theta)=\left(\begin{array}{cc}
0.5 & 0.5 e^{-i \theta} \\
0.5 & -0.5 e^{-i \theta}
\end{array}\right)
$$


and

$$
\mathbf{h}(\theta)=\left(\begin{array}{cc}
2(1+\cos (\theta)) & \sin (1)\left(e^{i 2 \theta}+e^{i \theta}\right) \\
\sin (1)\left(e^{-i 2 \theta}+e^{-i \theta}\right) & 2(1+\cos (1) \cos (\theta))
\end{array}\right) .
$$

By taking $T=2$, Figure 2 depicts the average distance between the actual spectral density matrix and its estimator for different choices of sample size.

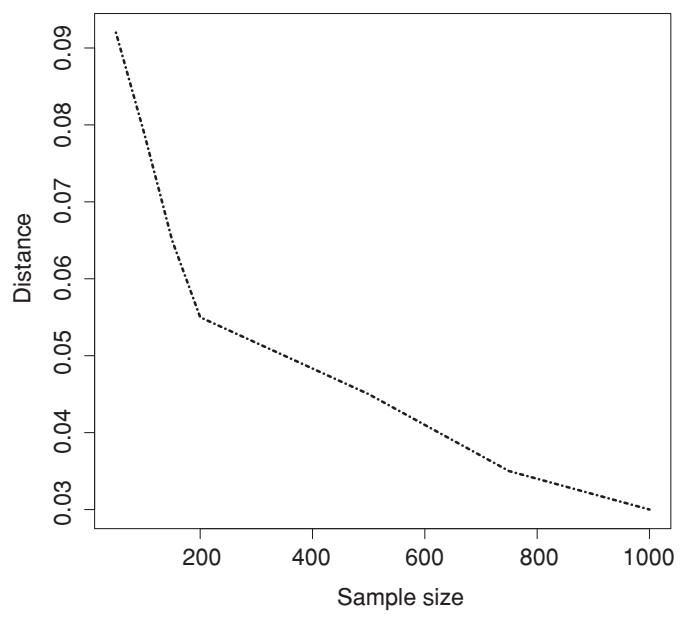

FiguRE 2 . The Chernoff distance ( $\alpha=0.1$ ) between actual spectral density matrix of the process (5.6) and its estimate for different choices of sample size.

EXAMPle 5.3. Assume a zero mean PC process $\left\{X_{t}, t \in \mathbb{Z}\right\}$ admits the representation

$$
X_{t}=g(t) Y_{t}
$$

where $g(t)$ is a periodic function with period $T$, and $Y_{t}$ is a stationary process. In general, the spectral components of this process are given by

$$
f_{k}(\theta)=\sum_{p=0}^{T-1} f_{Y}\left(\theta-\frac{2 \pi p}{T}\right) G_{p} \overline{G_{p-k}}, \quad k=0, \ldots, T-1,
$$

where $G_{p}=\frac{1}{T} \sum_{t=0}^{T-1} g(t) e^{-i \frac{2 \pi t p}{T}}$ and $f_{Y}(\theta)$ is the spectral density matrix of $Y_{t}$, see Hurd and Miamee [3].

To perform a numerical study, let us assume $g(t)=a(1+\cos (2 \pi t / T))$ and $Y_{t}$ is an $\operatorname{AR}(1)$ process, $Y_{t}=\phi Y_{t-1}+Z_{t}$. Figure 3 shows the average distance between the actual spectral density matrix and its estimator for different choices of sample size and period $T=4$.

Figures 1-3 evidently show the consistency of the weighted periodogram (4.7) to estimate the spectral density matrix of PC processes. 


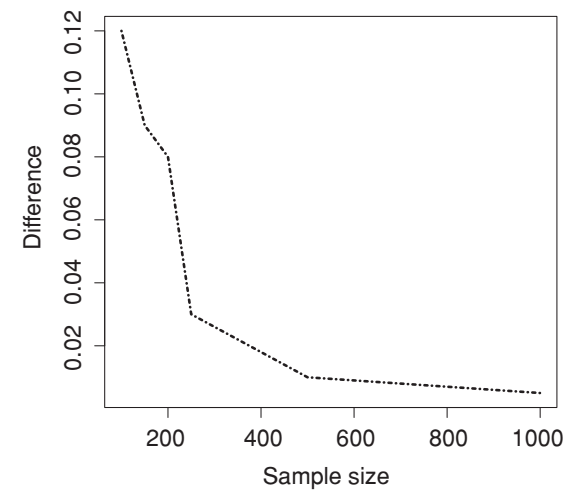

FiguRE 3 . The Chernoff distance $(\alpha=0.95)$ between actual spectral density matrix of the process (5.7) and its estimate by simulated data for different choices of sample size: $a=2$ and $\phi=0.8$.

\section{APPENDIX}

Pro of of Le m m a 3.1. (i) Following Proposition 10.1 in Brockwell and Davis [I]], we can conclude that

$$
\begin{aligned}
& \sigma_{i j}^{(Y)}(p, q ; s, r)= \\
& \begin{cases}\frac{-1}{N^{2}} D_{N}(2 \pi(p+s-q-r) / T), & p \neq q \neq s \neq r, \\
\left\{K_{N}\left(\lambda_{i}+\lambda_{j}+4 \pi p / T\right)-1\right\} / N, & p=q=s=r, \\
\left\{K_{N}\left(\lambda_{i}+\lambda_{j}+2 \pi(p+r) / T\right)\right. & \\
\left.+K_{N}\left(\lambda_{i}-\lambda_{j}+2 \pi(p-r) / T\right)-2\right\} / N, & (p=q) \neq(s=r), \\
\left\{K_{N}\left(\lambda_{i}+\lambda_{j}+2 \pi(p+s) / T\right)-1\right\} / N, & (p=r) \neq(s=q), \\
\left\{S_{N}\left(\lambda_{i}+\lambda_{j}, 4 \pi p / T, 4 \pi q / T\right)\right. & \\
\left.+K_{N}\left(\lambda_{i}-\lambda_{j}+2 \pi(p-r) / T\right)\right\} / N, & (p=s) \neq(r-q) .\end{cases}
\end{aligned}
$$

Without loss of generality suppose $\lambda_{i}<\lambda_{j}$ and also $\lambda_{i}^{(N)}, \lambda_{j}^{(N)}$ are two sequences of Fourier frequencies so that $\lambda_{i}^{(N)}<\lambda_{i}<\lambda_{j}^{(N)}<\lambda_{j}$, where $\lambda_{i}^{(N)} \rightarrow \lambda_{i}$ and $\lambda_{j}^{(N)} \rightarrow \lambda_{j}$. By replacing $\lambda_{i}$ and $\lambda_{j}$ with $\lambda_{i}^{(N)}$ and $\lambda_{j}^{(N)}$, respectively, and using the properties (iv) and (v) of $S_{N}(\cdot ; \cdot, \cdot)$ for large $N$, we can deduce that

$$
\sigma_{i j}^{(Y)}(p, q ; s, r)=O\left(N^{-1}\right) .
$$

This proves the statement (i) of Lemma 3.1. Other statements can be concluded by similar arguments.

Proof of Theorem 3.1. As in the proof of Lemma 3.1 suppose $\lambda_{i}^{(N)}<$ $\lambda_{i}<\lambda_{j}^{(N)}<\lambda_{j}$. Let us use the notation $\sigma_{i j}^{(\tilde{X})}(p, q ; s, r \| N)$ while $\lambda_{i}$ and $\lambda_{j}$ are 
replaced by $\lambda_{i}^{(N)}$ and $\lambda_{j}^{(N)}$. By using (2.7) we can write

$$
\begin{aligned}
\sigma_{i j}^{(\tilde{X})}(p, q ; s, r \| N)= & \sum_{k, k^{\prime}, l, l^{\prime}=0}^{T-1} a_{k}\left(\lambda_{i}^{(N)}+\frac{2 \pi p}{T}\right) \overline{a_{k^{\prime}}\left(\lambda_{i}^{(N)}+\frac{2 \pi q}{T}\right)} \\
& \times a_{l}\left(\lambda_{j}^{(N)}+\frac{2 \pi s}{T}\right) \overline{a_{l^{\prime}}\left(\lambda_{j}^{(N)}+\frac{2 \pi r}{T}\right)} \sigma_{i j}^{(Y)}(p, q ; s, r \| N) .
\end{aligned}
$$

Since $\lambda_{i}, \lambda_{j} \in[0,2 \pi / T)$, we can conclude by Lemma 3.1(i) that

$$
\begin{aligned}
\sigma_{i j}^{(\tilde{X})}(p, q ; s, r \| N)= & O\left(\frac{1}{N}\right) \sum_{k, k^{\prime}, l, l^{\prime}=0}^{T-1} a_{k}\left(\lambda_{i}^{(N)}+\frac{2 \pi p}{T}\right) \overline{a_{k^{\prime}}\left(\lambda_{i}^{(N)}+\frac{2 \pi q}{T}\right)} \\
& \times a_{l}\left(\lambda_{j}^{(N)}+\frac{2 \pi s}{T}\right) \overline{a_{l^{\prime}}\left(\lambda_{j}^{(N)}+\frac{2 \pi r}{T}\right)} \\
= & O\left(\frac{1}{N}\right) .
\end{aligned}
$$

In the case that $\lambda_{i}=\lambda_{j}$, suppose $\lambda_{i}^{(N)} \rightarrow \lambda_{i}$. Thus, by (2.7), we can write

$$
\begin{aligned}
\sigma_{i j}^{(\tilde{X})}(p, q ; s, r \| N)= & \sum_{i=1}^{3} \sum_{C_{i}} a_{k}\left(\lambda_{i}^{(N)}+\frac{2 \pi p}{T}\right) \overline{a_{k^{\prime}}\left(\lambda_{i}^{(N)}+\frac{2 \pi q}{T}\right)} a_{l}\left(\lambda_{i}^{(N)}+\frac{2 \pi s}{T}\right) \\
& \times \overline{a_{l^{\prime}}\left(\lambda_{i}^{(N)}+\frac{2 \pi r}{T}\right)} \sigma_{i j}^{(Y)}(p, q ; s, r \| N) \\
= & B_{1}+B_{2}+B_{3},
\end{aligned}
$$

where

$$
\begin{gathered}
C_{1}=\left\{\left(k, k^{\prime}, l, l^{\prime}\right) \in\{0, \ldots, T-1\}: p-k=q-k^{\prime}=s-l=r-l^{\prime}\right\}, \\
C_{2}=\left\{\left(k, k^{\prime}, l, l^{\prime}\right) \in\{0, \ldots, T-1\}:\left(p-k=r-l^{\prime}\right) \neq\left(q-k^{\prime}=s-l\right)\right\}, \\
C_{3}=\left\{\left(k, k^{\prime}, l, l^{\prime}\right) \notin C_{1} \cup C_{2}\right\} .
\end{gathered}
$$


According to Lemma 3.1(iii) and continuity of $a_{k}(\cdot)$, we can obtain

$$
\begin{aligned}
B_{1}= & {\left[1+O\left(\frac{1}{N}\right)\right] \sum_{C_{1}} a_{k}\left(\lambda_{i}+\frac{2 \pi p}{T}\right) \overline{a_{k^{\prime}}\left(\lambda_{i}+\frac{2 \pi q}{T}\right)} } \\
& \times a_{l}\left(\lambda_{i}+\frac{2 \pi s}{T}\right) \overline{a_{l^{\prime}}\left(\lambda_{i}+\frac{2 \pi r}{T}\right)} \\
= & {\left[1+O\left(\frac{1}{N}\right)\right] \sum_{k=0}^{T-1} a_{k}(\theta) a_{k+(q-p)}\left(\theta+\frac{2 \pi(q-p)}{T}\right) } \\
= & \sum_{k=0}^{T-1} a_{k}(\theta) a_{k+(q-p)}\left(\theta+\frac{2 \pi(q-p)}{T}\right) \\
& \times a_{k+(s-p)}\left(\theta+\frac{2 \pi(s-p)}{T}\right) \overline{a_{k+(r-p)}\left(\theta+\frac{2 \pi(r-p)}{T}\right)} \\
= & R(p, q ; s, r)+O\left(\frac{1}{N}\right),
\end{aligned}
$$

where $\theta=\lambda_{i}^{(N)}+2 \pi p / T$;

$$
\begin{aligned}
B_{2}= & \sum_{C_{2}} a_{k}\left(\lambda_{i}^{(N)}+\frac{2 \pi p}{T}\right) \overline{a_{k^{\prime}}\left(\lambda_{i}^{(N)}+\frac{2 \pi q}{T}\right)} a_{l}\left(\lambda_{i}^{(N)}+\frac{2 \pi s}{T}\right) \\
& \times a_{l^{\prime}}\left(\lambda_{i}^{(N)}+\frac{2 \pi r}{T}\right) \sigma_{i j}^{(Y)}(p, q ; s, r \| N) \\
= & {\left[1+O\left(\frac{1}{N}\right)\right] \sum_{C_{2}} a_{k}\left(\lambda_{i}^{(N)}+\frac{2 \pi p}{T}\right) \overline{a_{k^{\prime}}\left(\lambda_{i}^{(N)}+\frac{2 \pi q}{T}\right)} } \\
& \times a_{l}\left(\lambda_{i}^{(N)}+\frac{2 \pi s}{T}\right) \overline{a_{l^{\prime}}\left(\lambda_{i}^{(N)}+\frac{2 \pi r}{T}\right)} \\
= & \sum_{k \neq k^{\prime}} a_{k}\left(\lambda_{i}+\frac{2 \pi p}{T}\right) \overline{a_{k^{\prime}}\left(\lambda_{i}+\frac{2 \pi q}{T}\right)} a_{k^{\prime}}+(s-q)\left(\lambda_{i}+\frac{2 \pi s}{T}\right) \\
& \times a_{k+(r-p)}\left(\lambda_{i}+\frac{2 \pi r}{T}\right)+O\left(\frac{1}{N}\right) \\
= & {\left[\sum_{k=0}^{T-1} a_{k}(\theta) a_{k+(r-p)}\left(\theta+\frac{2 \pi(r-p)}{T}\right)\right] } \\
& \times\left[\sum_{k^{\prime}=0}^{T-1} \overline{a_{k^{\prime}}\left(\theta^{\prime}\right)} a_{k^{\prime}+(s-q)}\left(\theta^{\prime}+\frac{2 \pi(s-q)}{T}\right)\right]-R(p, q, r, s)+O\left(\frac{1}{N}\right),
\end{aligned}
$$


where $\theta^{\prime}=\lambda_{i}+2 \pi q / T$

$$
\begin{aligned}
B_{3}=\left[O\left(\frac{1}{N}\right)\right] \sum_{C_{3}} & a_{k}\left(\lambda_{i}^{(N)}+\frac{2 \pi p}{T}\right) \overline{a_{k^{\prime}}\left(\lambda_{i}^{(N)}+\frac{2 \pi q}{T}\right)} \\
\times & a_{l}\left(\lambda_{i}^{(N)}+\frac{2 \pi s}{T}\right) \overline{a_{l^{\prime}}\left(\lambda_{i}^{(N)}+\frac{2 \pi r}{T}\right)}=O\left(\frac{1}{N}\right) .
\end{aligned}
$$

Therefore, we obtain

$$
\begin{aligned}
\sigma_{i j}(p, q ; s, r)= & {\left[\sum_{k=0}^{T-1} a_{k}(\theta) \overline{a_{k+(r-p)}\left(\theta+\frac{2 \pi(r-p)}{T}\right)}\right] } \\
& \times\left[\sum_{k^{\prime}=0}^{T-1} \overline{a_{k^{\prime}}\left(\theta^{\prime}\right)} a_{k^{\prime}+(s-q)}\left(\theta^{\prime}+\frac{2 \pi(s-q)}{T}\right)\right] \\
& -R(p, q, r, s)+R(p, q, r, s)+O\left(\frac{1}{N}\right) \\
= & f_{r-p}\left(\lambda_{i}+\frac{2 \pi p}{T}\right) \overline{f_{s-q}\left(\lambda_{i}+\frac{2 \pi q}{T}\right)}+O\left(\frac{1}{N}\right) .
\end{aligned}
$$

Pro of of The or e m 4.1. (a) It is immediate from (4.4) and the continuity of $\mathbf{f}(\cdot)$ that

$$
E\left(\tilde{\mathbf{f}}\left(\lambda_{j}\right)\right)=E\left(\sum_{|k| \leqslant u_{n}} W_{n}(k) \tilde{\mathbf{I}}\left(\lambda_{j}+\lambda_{k}\right)\right)=\mathbf{f}\left(\lambda_{j}\right) \sum_{|k| \leqslant u_{n}} W_{n}(k)=\mathbf{f}\left(\lambda_{j}\right) .
$$

(b) Using (4.6), we get

$$
\operatorname{cov}\left(\tilde{f}_{p q}\left(\lambda_{i}\right), \tilde{f}_{s r}\left(\lambda_{j}\right)\right)=\sum_{|g|,|t| \leqslant m} W_{n}(g) W_{n}(t) \operatorname{cov}\left(\tilde{I}_{p q}\left(\lambda_{i}+\lambda_{g}\right), \tilde{I}_{s r}\left(\lambda_{j}+\lambda_{t}\right)\right) .
$$

If $\lambda_{i} \neq \lambda_{j}, \lambda_{i}+\lambda_{j} \neq 2 \pi / T$ and $N$ is sufficiently large, then $\lambda_{i}+\lambda_{g} \neq \lambda_{j}+\lambda_{t}$ for all $|g|,|t| \leqslant u_{n}$. Therefore, using Corollary 3.1, we can obtain

$$
\begin{aligned}
\left|\operatorname{cov}\left(\tilde{f}_{p q}\left(\lambda_{i}\right), \tilde{f}_{s r}\left(\lambda_{j}\right)\right)\right| & =\left|\sum_{|g| \leqslant m|t| \leqslant m} \sum_{n}(g) W_{n}(t) O\left(N^{-1}\right)\right| \\
& \leqslant O\left(\frac{1}{N}\right)\left(\sum_{|t| \leqslant m} W_{n}(t)\right)^{2} .
\end{aligned}
$$

It follows from (4.5)) that the covariance approaches zero.

Also, in the case that $\lambda_{i}+\lambda_{j}=2 \pi / T$, we have

$\operatorname{cov}\left(\tilde{f}_{p q}\left(\lambda_{i}\right), \tilde{f}_{s r}\left(\lambda_{j}\right)\right)=\operatorname{cov}\left(\sum_{|g| \leqslant u_{n}} W_{n}(g) \tilde{I}_{p q}\left(\lambda_{i}+\lambda_{g}\right), \sum_{|t| \leqslant u_{n}} W_{n}(t) \tilde{I}_{s r}\left(\lambda_{j}+\lambda_{t}\right)\right)$. 
Let $D_{1}=\{(t, g):|t|+|g|=m l\}$ and $D_{2}=\{(t, g):|t|+|g| \neq m l\}$. Using Lemma 3.1(ii), we can conclude that

$$
\begin{aligned}
\operatorname{cov}\left(\tilde{f}_{p q}\left(\lambda_{i}\right), \tilde{f}_{s r}\left(\lambda_{j}\right)\right)= & B\left(\lambda_{i}\right)\left(1-O\left(N^{-1}\right)\right) \sum_{D_{1}} W_{n}(t) W_{n}(g) \\
& +O\left(N^{-1}\right) \sum_{D_{2}} W_{n}(t) W_{n}(g)
\end{aligned}
$$

where

$$
\begin{aligned}
B\left(\lambda_{i}\right)= & {\left[\sum_{k} a_{k}(\theta) a_{p+s-(T-1)-k}\left(\theta-\frac{2 \pi}{T}(p+s-(T-1))\right)\right] } \\
& \times\left[\sum_{k^{\prime}} \overline{a_{k^{\prime}}\left(\theta^{\prime}\right)} \overline{a_{q+r-(T-1)-k^{\prime}}\left(\theta^{\prime}-\frac{2 \pi}{T}(q+r-(T-1))\right)}\right]
\end{aligned}
$$

and $\theta=\lambda_{i}+2 \pi p / T, \theta^{\prime}=\lambda_{i}+2 \pi q / T$. Since for large $N, \lambda_{k}+\lambda_{j} \rightarrow 0$, we have $D_{1} \rightarrow \emptyset$. Thus we conclude that

$$
\operatorname{cov}\left(\tilde{f}_{p q}\left(\lambda_{i}\right), \tilde{f}_{s r}\left(\lambda_{j}\right)\right) \rightarrow 0 .
$$

(c) We note that

$$
\begin{aligned}
\operatorname{cov}\left(\tilde{f}_{p q}\left(\lambda_{i}\right), \tilde{f}_{s r}\left(\lambda_{i}\right)\right) \\
=\left(\sum_{|t| \leqslant m} W_{n}^{2}(t)\right)\left(f_{r-p}\left(\lambda_{i}+\frac{2 \pi p}{T}\right) \overline{f_{s-q}\left(\lambda_{i}+\frac{2 \pi q}{T}\right)}+O\left(N^{-1}\right)\right) \\
\quad+\sum_{g \neq t} W_{n}(g) W_{n}(t) O\left(N^{-1}\right) \\
=\left(\sum_{|t| \leqslant m} W_{n}^{2}(t)\right) f_{r-p}\left(\lambda_{i}+\frac{2 \pi p}{T}\right) \overline{f_{s-q}\left(\lambda_{i}+\frac{2 \pi q}{T}\right)} \\
\quad+O\left(\frac{1}{N}\right) O\left(\sum_{|t| \leqslant m} W_{n}^{2}(t)\right)+O\left(\frac{1}{N}\right)\left(\sum_{|t| \leqslant m} W_{n}^{2}(t)\right)(2 m+1) .
\end{aligned}
$$

According to the properties (4.2), (4.4), and (4.5)), all term tends to zero for $N$ sufficiently large. Moreover, in this case,

$$
\left(\sum_{|t| \leqslant m} W_{n}^{2}(t)\right)^{-1} \operatorname{cov}\left(\hat{f}_{p q}\left(\lambda_{i}\right), \hat{f}_{s r}\left(\lambda_{i}\right)\right) \rightarrow f_{r-p}\left(\lambda_{i}+\frac{2 \pi p}{T}\right) \overline{f_{s-q}\left(\lambda_{i}+\frac{2 \pi q}{T}\right)}
$$

giving (d). 


\section{REFERENCES}

[1] P. J. Brockwell and R. A. Davis, Time Series: Theory and Methods, second edition, Springer, New York 1991.

[2] E. G. Gladyshev, Periodically correlated random sequences, Soviet Math. Dokl. 2 (1961), pp. 385-388.

[3] H. L. Hurd and A. G. Miamee, Periodically Correlated Random Sequences: Spectral Theory and Practice, Wiley, Hoboken, NJ, 2007.

[4] Y. Kakizawa, R. H. Shumway, and M. Taniguchi, Discrimination and clustering for multivariate time series, J. Amer. Statist. Assoc. 93 (1998), pp. 328-340.

[5] A. L. Khuri, T. Mathew, and D. G. Nel, A test to determine closeness of multivariate Satterthwaite's approximation, J. Multivariate Anal. 51 (1) (1994), pp. 201-209.

[6] A. R. Nematollahi and S. Rao, On the spectral density estimation of periodically correlated time series, Sankhya 67 (3) (2005), pp. 568-589.

[7] A. R. Soltani and M. Azimmohseni, Periodograms asymptotic distributions in periodically correlated processes and multivariate stationary processes: An alternative approach, J. Statist. Plann. Inference 137 (2007), pp. 1236-1242.

[8] A. R. Soltani and Z. Shishebor, Weakly periodic sequences of bounded linear transformation, Georgian Math. J. 6 (1) (1999), pp. 91-98.

[9] W. Y. Tan and R. P. Gupta, On approximating a linear combination of central Wishart matrices with positive coefficients, Comm. Statist. Theory Methods 12 (22) (1983), pp. 2589 2600.

Majid Azimmohseni

Department of Statistic, Faculty of Science

Golestan University

P.O. Box 155, Gorgan, Iran

E-mail:m.azim@gu.ac.ir

Mahnaz Khalafi

Department of Statistic, Faculty of Science

Golestan University

P.O. Box 155, Gorgan, Iran

E-mail: m.khalafi@gu.ac.ir
Ahmad Reza Soltani Department of Statistics and Operations Research Faculty of Science, Kuwait University P.O. Box 5969, Safat 13060, Kuwait E-mail: Soltani@kuc01.kuniv.edu.kw

Naeemeh Akbari Ghalesary Department of Statistic, Faculty of Science Golestan University P.O. Box 155, Gorgan, Iran E-mail:edi.lord.65@gmail.com

Received on 2.12.2016;

revised version on 16.1.2017 